\title{
New hope for Alzheimer's dementia as prospects for disease modification fade: symptomatic treatments for agitation and psychosis
}

\author{
Stephen M. Stahl \\ ISSUE: \\ More than a decade of failure to find disease-modifying treatments for Alzheimer's \\ dementia has driven the field back to finding better symptomatic treatments for \\ behavioral symptoms of dementia, especially psychosis and agitation.
}

\section{Take-Home Points}

- Attempts to halt or reverse the degenerative process in Alzheimer's dementia have targeted beta amyloid.

- Although numerous mechanisms to reduce beta amyloid have been successful in engaging this target, none has yet modified the course of Alzheimer's dementia.

- While the field sorts out whether to target beta amyloid, tau, or other potential neurodegenerative mechanisms, the epidemic of Alzheimer's dementia rages on, with an exploding population of patients who not only have progressive neurodegenerative dementia with cognitive and memory problems, but also common and severe behavioral symptoms including agitation and psychosis.

- Several novel therapeutics for Alzheimer's dementia are now focusing on symptomatic treatments for agitation and psychosis while the search for disease modifying treatments retrenches and looks for new targets.

\section{Introduction}

The bad news is that without disease modifying treatments, the incidence of dementia in the United States is projected to skyrocket from about 5 million individuals affected today to almost 14 million by 2030, all of whom will have disabling behavioral symptoms, from memory disturbances to agitation to psychosis. ${ }^{1}$ The further bad news is that setbacks in testing amyloid modifying treatments that successfully target beta amyloid do not yet appear to be disease modifying for Alzheimer's dementia, ${ }^{2,3}$ which means that the explosion of cases will continue unabated. The good news is that new pharmacologic approaches to treating the behavioral symptoms of dementia, which will accompany those who have dementia until disease modifying treatments are discovered, have shown good promise, particularly in the area of agitation and psychosis; these will be reviewed here.

Although treatment options limited to symptomatic presentations of the behavioral and neuropsychiatric symptoms of dementia do not arrest or reverse the course of dementia, they are nevertheless useful and strive to relieve patients and caregivers of some of the behavioral and psychiatric sequelae that can severely impact their quality of life. In fact, neuropsychiatric symptoms of dementia affect virtually all patients with dementia at some time during the disease course and are associated with earlier institutionalization, increased caregiving costs (both financial and otherwise), and worsened disease progression. ${ }^{4}$ Unfortunately, pharmacological measures acting on neurocircuitry that may be damaged in the brains of patients with dementia as well as attempts to utilize nonpharmacological, cognitionacting strategies in cognitively impaired patients with dementia may not be as effective as they are for younger, non-demented patients. It is also important to note that improvements in behavioral symptoms may not be due solely to a prescribed psychotropic agent but may also be a result of the extra social contact with the treatment 


\section{BRAINSTORMS-Clinical Neuroscience Update}

team that medication warrants. In fact, current first-line treatment of any behavioral symptom associated with dementia is nonpharmacological. ${ }^{4}$ However, nonpharmacological interventions are frequently inadequate and have spurred the search for symptomatic treatments while the search for disease-modifying therapies retrenches.

\section{What Are the Behavioral Symptoms of Dementia, and How Are They Currently Treated?}

There are many behavioral symptoms associated with dementia (Figure 1), ${ }^{4}$ and the Neuropsychiatric Inventory Questionnaire (NPI) is an excellent resource for evaluating not only the range and severity of a variety of secondary behavioral symptoms associated with dementia but also the impact that such behaviors have on the caregiver. 5,6 For each behavior, severity is rated on a scale of $1-3$ (with 1 being mild), and caregiver distress is rated on a scale of $0-5$ (with 0 being not distressing at all)., ${ }^{5,6}$ To the extent that there are any pharmacological treatments for the behavioral symptoms of dementia, they are all relatively nonspecific and often poorly evidence-based and off-label. ${ }^{4}$ In fact, one of the most powerful pharmacologic treatments of dementia

Figure 1. The Neuropsychiatric Inventory Questionnaire (NPI) is an excellent resource for evaluating not only the severity of a variety of secondary behavioral symptoms associated with dementia but also the impact that such behaviors have on the caregiver. For each behavior, severity is rated on a scale of $1-3$ (with 1 being mild) and caregiver distress is rated on a scale of $0-5$ (with 0 being not distressing at all).

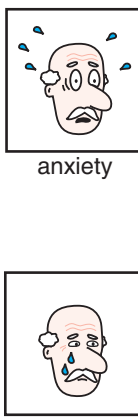

depression

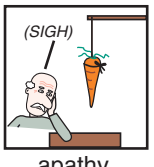

$$
\text { apathy }
$$
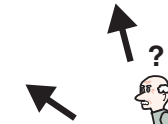

?
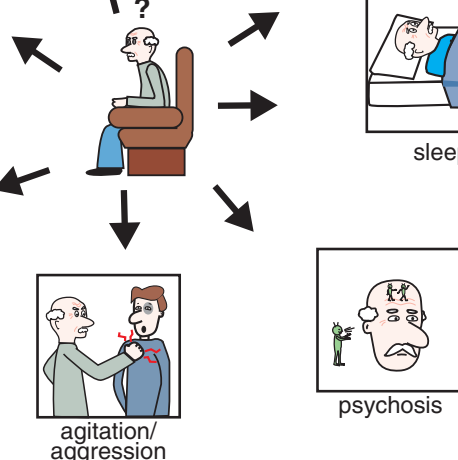

psychosis
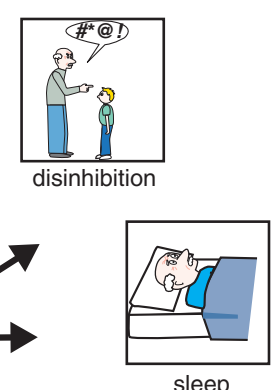

sleep

currently is to discontinue drugs causing worsening of behavioral symptoms often through unwanted drug interactions. ${ }^{4}$ It has been estimated that approximately $60 \%$ of nursing home residents (undoubtedly many of whom have dementia) are prescribed unnecessary medications, whereas $42 \%$ may not be getting potentially beneficial medications. ${ }^{4}$ This failure to properly utilize medications in the elderly population contributes to morbidity, mortality, and poor quality of life. Additionally, many geriatric patients, including those with dementia, are on numerous medications for a variety of physical, neurological, and psychological disorders. It is critical that potential drug interactions are considered before placing an elderly patient with dementia on a new medication, as the consequences of certain drug interactions can be fatal.

\section{Psychosis and Dementia}

Symptoms of psychosis, including hallucinations and delusions, affect many individuals with varying types of dementia, especially in later stages of disease course (Table 1). ${ }^{4}$ Although psychotic symptoms are often episodic and may resolve during the course of dementia, they can be quite troubling for caregivers and may increase the chance of institutionalization. In Alzheimer's disease, delusions may be more common than hallucinations, whereas, in dementia with Lewy bodies, hallucinations are more common. Psychotic symptoms seem to be related to pathology in the neocortex, and specific symptoms (eg, visual vs auditory hallucinations) likely reflect damage to specific cortical areas. ${ }^{4}$

Conventional, first-generation antipsychotics (such as haloperidol) and the atypical, second-generation antipsychotics risperidone, olanzapine, quetiapine, and aripiprazole (all of which block dopamine D2 receptors)

Table 1. Symptoms of psychosis, including hallucinations and delusions, affect many individuals with varying types of dementia, especially in later stages of disease course $e^{4}$. Although psychotic symptoms are often episodic and may resolve during the course of dementia, they can be quite troubling for caregivers and may increase the chance of institutionalization.

Prevalence of psychosis

Alzheimer's disease $25-40 \%$

Dementia with Lewy bodies $75 \%$

Parkinson's disease dementia $50-60 \%$

Frontotemporal dementia $5-10 \%$

Vascular dementia $15 \%$ 


\section{BRAINSTORMS-Clinical Neuroscience Update}

Figure 2. (A) According to the dopamine D2 hypothesis of psychosis, dopaminergic projections along the mesolimbic pathway (blue; from the ventral tegmental area [VTA] to the ventral striatum) are hyperactive, leading to excess dopaminergic neurotransmission in the ventral striatum and resulting in psychosis, particularly delusions and auditory hallucinations. On the other hand, as neuropathology such as Lewy bodies leads to death of dopaminergic neurons of the nigrostriatal pathway, there is a paucity of dopaminergic input to the dorsal striatum, resulting in movement symptoms. Treatment with a dopamine D2 receptor antagonist such as an antipsychotic can act in the ventral striatum to reduce dopaminergic input, thereby ameliorating symptoms of psychosis. However, the antipsychotic blockade of dopamine D2 receptors in the dorsal striatum may actually further reduce the already limited dopaminergic input from the substantia nigra, leading to worsening of motor symptoms. (B) According to the glutamatergic NMDA hypothesis of psychosis, within the cerebral cortices (where amyloid, tau, or Lewy body neuropathology may be prevalent), (1) glutamatergic input to GABA interneurons is reduced due to hypoactive NMDA receptors located on GABA neurons. These GABA neurons are therefore not activated, leading to (2) reduced GABAergic input on downstream glutamatergic neurons, i.e. disinhibition of (3) glutamatergic input onto, and thus activation of, dopaminergic neurons in the VTA occurs, leading to (4) excessive dopaminergic input to the ventral striatum and psychosis. (C) The serotonin 5HT2A hypothesis of psychosis posits that loss of serotonergic neurons in the raphe nuclei (due to Lewy body, amyloid, and/or tau pathology) leads to (1) hypoactive serotonergic neurotransmission in the cerebral cortices, which hypothetically causes upregulation of serotonin 5HT2A receptors located on glutamatergic neurons. This excessive glutamate, particularly in visual cortices, is thought to be the neurobiological substrate for visual hallucinations as are often seen in Parkinson's disease psychosis. (2) Glutamatergic input onto, and thus activation of, dopaminergic neurons in the VTA also occurs, leading to (3) excessive dopaminergic input to the ventral striatum and psychosis.

(a) Dopamine hypothesis of psychosis

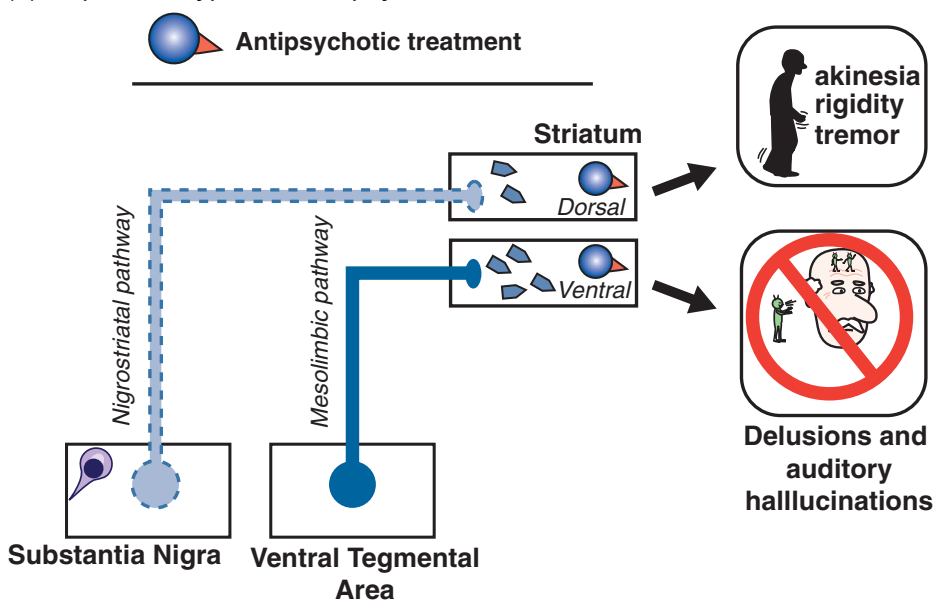

(b) Glutamate hypothesis of psychosis

Visual, temporal, motor and prefrontal cortices
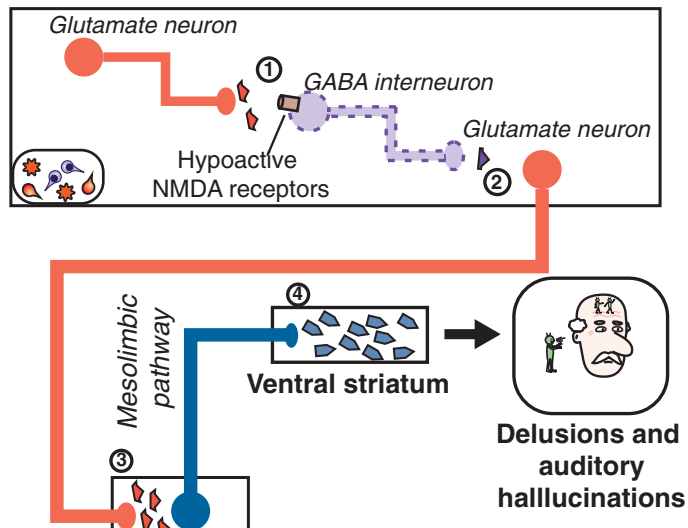

(c) Serotonin hypothesis of psychosis

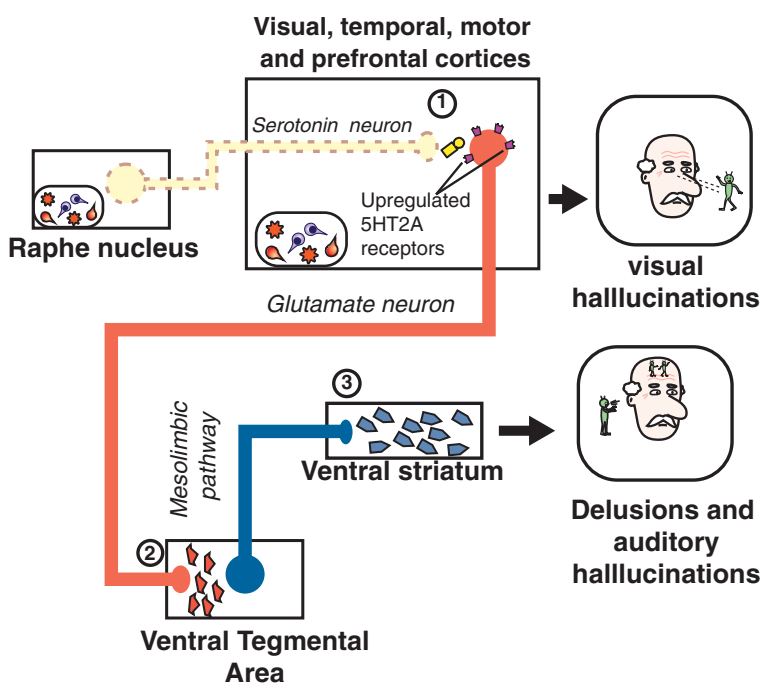




\section{BRAINSTORMS-Clinical Neuroscience Update}

are commonly used off label and with considerable controversy to treat psychosis (and agitation) in patients with dementia. ${ }^{4}$ However, these agents may have limited efficacy and come with a wealth of side-effect risks including weight gain and other cardiometabolic issues, anticholinergic-associated worsening of cognition, and sedation, as well as inducing or worsening movement disorders via antagonism at dopamine $\mathrm{D} 2$ receptors in the dorsal striatum-which may worsen motor symptoms in patients with Parkinson's disease and other dementia types in which motor symptoms prevail. Importantly, the use of antipsychotics in elderly patients with dementia has been associated with an increased risk of mortality, warranting an FDA black box warning against their use in this population. For these reasons, it is recommended that current antipsychotics only be used in cases where psychotic behavior is severe, potentially dangerous, and at the lowest dose possible for the shortest duration feasible. ${ }^{4}$ So, how can a novel treatment reduce psychosis in dementia without D2 blockade and without these risks?

The answer may be to target something other than D2 dopamine receptors in psychosis associated with dementia. We have recently reviewed the 3 major theories of psychosis, ${ }^{7}$ which hypothesized to result not just from hyperactive dopamine at D2 receptors in the mesolimbic pathway (Figure 2A), but 2 other theories suggest hyperactive serotonin (5HT, 5-hydroxytryptamine) at 5HT2A receptors in the cortex (Figure 2B) and/or hypoactive glutamate at NMDA (N-methyl-daspartate) glutamate receptors in the cortex (Figure 2C). ${ }^{4,7}$ It is entirely possible that more than one of these dysfunctional circuits are the cause of psychosis in patients with dementia, especially as the underlying dementia-related pathology progresses and more brain areas become involved. ${ }^{4}$ Nevertheless, targeting either the glutamate or the serotonin pathways may be helpful in reducing the psychosis associated with dementia. Furthermore, these approaches may avoid the side effects and risks associated with D2 dopamine antagonists for psychosis.

Evidence that this approach may indeed be useful for a novel treatment of psychotic behavioral symptoms of dementia is the finding that the first non-D2 antagonist approved for the treatment of psychosis targets $5 \mathrm{HT} 2 \mathrm{~A}$ receptors. $^{8}$ That is, the selective $5 \mathrm{HT} 2 \mathrm{~A} / 2 \mathrm{C}$ antagonist pimavanserin (Figure 3)-which does not have D2 antagonist properties-improves psychosis associated with Parkinson's disease. ${ }^{8}$ Pimavanserin may be particularly effective in reducing visual hallucinations without exacerbating motor effects. Side effects may include peripheral edema, confusion, nausea, and potential QTC prolongation. ${ }^{8}$
Figure 3. Pimavanserin, a novel serotonin 5HT2A and 5HT2C antagonist with no dopamine D2 binding affinity, is now approved for the treatment of psychosis in Parkinson's disease and appears to be effective in reducing visual

hallucinations, without exacerbating motor effects. Side effects may include peripheral edema, confusion, nausea, and potential QTc prolongation. Pimavanserin is currently in testing for psychosis associated with other forms of dementia, such as Alzheimer's disease or all-cause dementia.

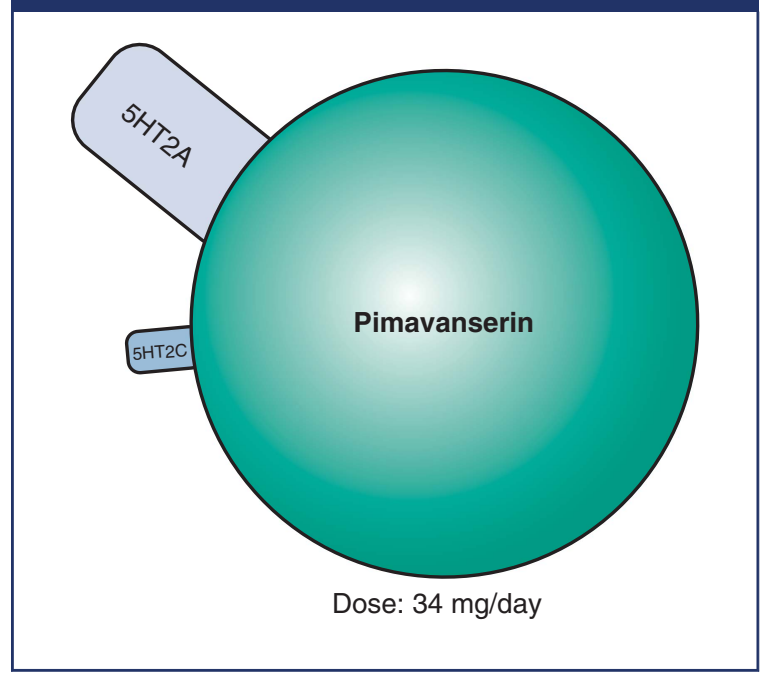

More recently, a Phase II trial reports that pimavanserin also improves the psychosis associated with Alzheimer's disease. ${ }^{9}$ Also, a Phase III study of psychosis from all causes of dementia is also now underway to determine whether pimavanserin can reduce psychotic symptoms and maintain this improvement in these patients. If so, pimavanserin would become not only the first non-D2 antagonist for the treatment of psychosis in dementia, but also in fact the first and only approved treatment of any kind for psychosis in dementia.

\section{Agitation and Dementia}

Agitation (encompassing emotional stress, excessive psychomotor activity, aggression, irritability, and disinhibition) is quite common in patients with dementia, affecting $50 \%$ or more of patients with Alzheimer's disease. ${ }^{4}$ First-line treatment involves addressing potential unmet needs that may be causing agitation, such as pain, hunger, or thirst. ${ }^{4}$ Agitation can be conceptualized as physical and verbal behaviors that are excessive, inappropriate, repetitive, nonspecific, and observable. The Cohen-Mansfield Agitation Inventory is a 29-item, clinician-rated, 7-point scale that can be used to assess both aggressive and non-aggressive 


\section{BRAINSTORMS - Clinical Neuroscience Update}

Table 2. Agitation can be conceptualized as physical and verbal behaviors that are excessive, inappropriate, repetitive, nonspecific, and

observable. The Cohen-Mansfield Agitation Inventory is a 29-item, clinician-rated, 7-point scale that can be used to assess both

aggressive and non-aggressive verbal and physical behaviors indicating agitation.

Cohen-Mansfield Agitation Inventory (CMAI)

\begin{tabular}{ll}
\hline Physical/Aggressive & Physical/Non-Aggressive \\
Hitting & Pacing, aimless wandering \\
Kicking & Inappropriate dress/disrobing \\
Grabbing & Trying to get to a different place \\
Pushing & Intentional falling \\
Throwing things & Eating/drinking inappropriate substances \\
Biting & Handling things inappropriately \\
Scratching & Hiding things \\
Spitting & Hoarding things \\
Hurting self or others & Performing repetitive mannerisms \\
Destroying property & General restlessness \\
Making physical sexual advances & \\
Verbal/Aggressive & \\
Screaming & Verbal/Non-Aggressive \\
Making verbal sexual advances & Repetitive sentences or questions \\
Cursing or verbal aggression & Strange noises \\
& Complaining \\
& Negativism
\end{tabular}

verbal and physical behaviors indicating agitation (Table 2). ${ }^{4}$

Agitation in patients with dementia may hypothetically stem from either impaired top-down cortical control of impulsive behaviors, irregular bottom-up drive from limbic regions, or as a result of psychosis. ${ }^{10,11}$ The neurotransmitters dopamine (DA), norepinephrine $(\mathrm{NE})$, serotonin $(5 \mathrm{HT})$, acetylcholine $(\mathrm{ACh})$, glutamate (Glu), and gamma-aminobutyric acid (GABA) and possibly sigma receptors are all thought to be involved in aggressive or agitated behaviors in the prefrontal cortex (PFC) of aggressive/agitated patients. ${ }^{10,11}$

According to published expert consensus guidelines for the pharmacological treatment of violence in association with cognitive impairment, treatment of agitation and aggression in patients with dementia, targeting neurotransmitters to hypothetically enhance top-down cortical control, reduce bottom-up limbic drives, or both, first involves non-antipsychotic-based therapies (eg, anticonvulsants, adrenergic agents, antidepressants), with next-line measures including antipsychotic treatment avoiding antipsychotics with a greater propensity for motor side effects in patients with Parkinson's disease dementia or other dementia types in which movement symptoms prevail. ${ }^{12}$
Figure 4. As a dopamine D2 receptor partial agonist, with dopamine D3, serotonin 5HT1A, serotonin 5HT2A, and adrenergic alpha- 1 receptors binding properties, the atypical antipsychotic brexpiprazole shares many properties with aripiprazole. Brexpiprazole is currently being tested for its efficacy and safety in the treatment of agitation in patients with Alzheimer's disease.

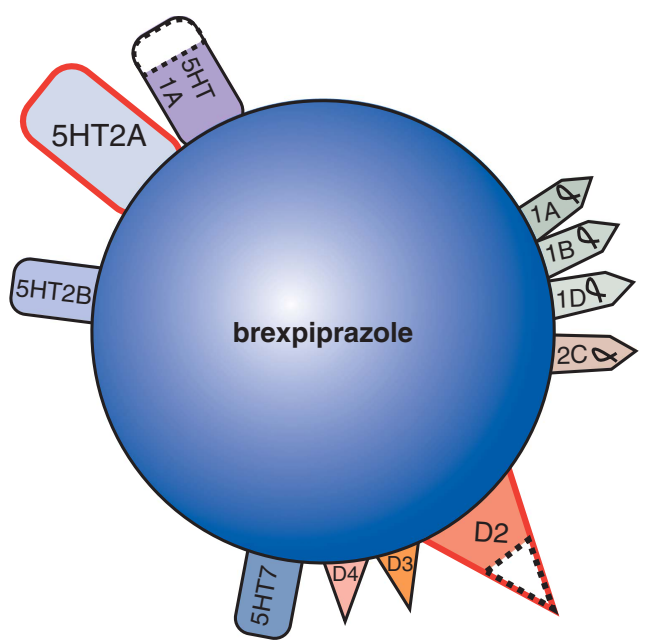




\section{BRAINSTORMS-Clinical Neuroscience Update}

New developments in the treatment of agitation in Alzheimer's dementia include evaluation of agents with novel therapeutic mechanisms in clinical trials utilizing well-defined and well-rated agitation (Table 2) in patients with Alzheimer's dementia. The novel dopamine 2 partial agonist brexpiprazole with multiple 5HT and alpha adrenergic receptor binding properties (Figure 4$)^{13}$ has reported evidence of efficacy in reducing agitation in Alzheimer's disease. ${ }^{14,15}$ The actions of brexpiprazole, although classified as an antipsychotic because it exhibits D2 properties, may be due to the non D2 binding properties of this agent (Figure 4), and the safety profile for cardiovascular, metabolic, and movement risk may be more acceptable than other agents in this class. ${ }^{14,15}$ Further studies will determine whether this agent will be heading for formal FDA approval for the treatment of agitation in Alzheimer's dementia.

Another set of developments are trials of the sigma and glutamate active agent dextromethorphan (combined with a CYP450 2D6 inhibitor) (Figure 5) Dextromethorphan (DXM) is a sigma-1 and mu opioid receptor agonist with antagonist properties at NMDA and nicotinic $\alpha 3 \beta 4$ receptors and inhibition of both serotonin and norepinephrine reuptake (SERT and NET, respectively). ${ }^{16}$ Dextromethorphan combined with quinidine $(\mathrm{Q})$, which inhibits the cytochrome P450 2D6 (CYP 2D6) enzyme that metabolizes DXM, thereby increasing the bioavailability of DXM 20-fold, is FDA-approved for the treatment of pseudobulbar affect. ${ }^{16}$ The rationale for possible efficacy in agitation of Alzheimer's disease derives from the finding that pseudobulbar affect is a condition that has been linked to the emotional instability of agitation in Alzheimer's disease. ${ }^{17}$ Recent data have indeed indicated that dextromethorphan-quinidine may significantly reduce agitation in patients with Alzheimer's disease with relatively good tolerability. ${ }^{18,19}$ Also, AVP-786, a deuterated version of DXM combined with a low dose of quinidine, is undergoing similar clinical testing.

Figure 5. Dextromethorphan (DXM) is a sigma-1 and mu opiate receptor agonist with antagonist properties at NMDA and nicotinic $\alpha 3 \beta 4$ receptors and inhibition of both serotonin and norepinephrine reuptake inhibitors (SERT and NET, respectively). Dextromethorphan combined with quinidine $(Q)$, which inhibits the cytochrome P450 2D6 (CYP 2D6) enzyme that metabolizes DXM, thereby increasing the bioavailability of DXM 20-fold, is FDA-approved for the treatment of pseudobulbar affect. Recent data have indicated that dextromethorphan-quinidine may reduce agitation in patients with Alzheimer's disease with relatively good tolerability. AVP-786, a deuterated version of DXM combined with a low dose of quinidine, is now in testing for agitation in Alzheimer dementia. Deuteration of dextromethorphan reduces first pass liver metabolism, slowing the rate of metabolism of dextromethorphan and allowing for an even lower dose of quinidine. Another combination of dextromethorphan with the antidepressant bupropion, a norepinephrine and dopamine reuptake inhibitor and nicotinic acetylcholine receptor antagonist that has CYP 2D6-blocking capabilities and thus inhibits metabolism of dextromethorphan, called AXS-05, is also being tested in agitation in Alzheimer's dementia.
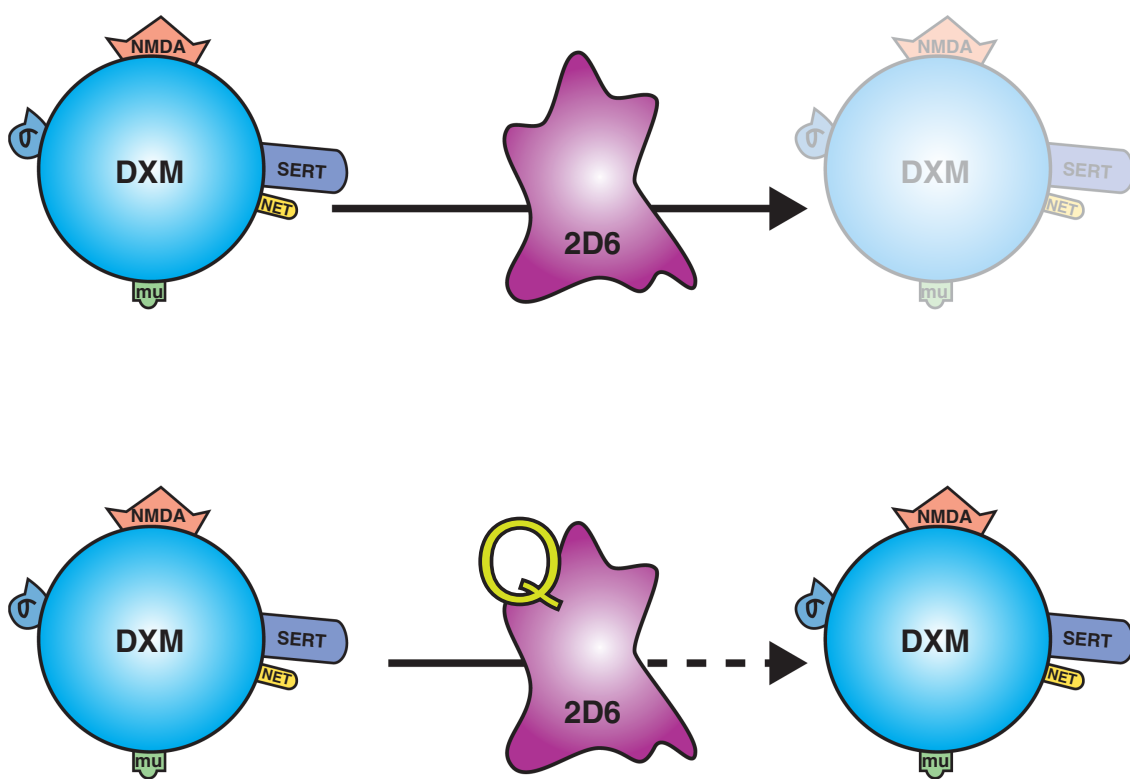


\section{BRAINSTORMS-Clinical Neuroscience Update}

Deuteration of dextromethorphan reduces first-pass liver metabolism, slowing the rate of metabolism of dextromethorphan and allowing for an even lower dose of quinidine. Yet another dextromethorphan formulation is in testing for agitation in Alzheimer's dementiaa combination of dextromethorphan with the antidepressant bupropion, a norepinephrine and dopamine reuptake inhibitor and nicotinic acetylcholine receptor antagonist that has CYP 2D6-blocking capabilities and thus inhibits metabolism of dextromethorphan and known as AXS-05. It will be interesting to see if either or both of these agents containing dextromethorphan gains FDA approval for agitation in Alzheimer's dementia, and whether the additional pharmacologic properties of bupropion are a positive factor in clinical outcome, or eventually, whether a combination of the different pharmacologic mechanisms of dextromethorphan/2D6 inhibition added to those of brexpiprazole would be even more robust in reducing agitation in dementia.

\section{References:}

1. Alzheimer's Association. Alzheimer's disease facts and figures. Alzheimers Dement. 2016;12(4):459-509.

2. Godyń J, Jończyk J, Panek D, et al. Therapeutic strategies for Alzheimer's disease in clinical trials. Pharmacol Res. 2016;68 (1):127-138.

3. Panza F, Seripa D, Solfrizzi V, et al. Emerging drugs to reduce abnormal $\beta$-amyloid protein in Alzheimer's disease patients. Expert Opin Emerg Drugs. 2016;21(4):377-391.

4. Stahl SM, Morrissette DA. Stahl's Illustrated Alzheimer's Disease and Other Dementias. Cambridge: Cambridge University Press; 2018.

5. Lanctôt KL, Amatniek J, Ancoli-Isreal S, et al. Neuropsychiatric signs and symptoms of Alzheimer's disease: new treatment paradigms. Alzheimers Dement (N Y). 2017;3(3):440-449.

6. Neuropsychiatric Inventory: setting the standard for Alzheimer research. http://npitest.net/. Accessed March 1, 2018.
7. Stahl SM. Beyond the dopamine hypothesis of schizophrenia to three neural networks of psychosis: dopamine, serotonin and glutamate. CNS Spectr. 2018;23(3):187-191.

8. Stahl SM. Mechanism of action of pimavanserin in Parkinson's disease psychosis: targeting serotonin 5HT2A and 5HT2C receptors. CNS Spectr. 2016;21(4):271-275.

9. Ballard C. Clinical trial of pimavanserin in Alzheimer psychosis. Abstract presented at the Clinical Trials for Alzheimer Disease meeting, Boston, November 2017.

10. Stahl SM. Deconstructing violence as a medical syndrome: mapping psychotic, impulsive, and predatory subtypes to malfunctioning brain circuits. CNS Spectr. 2014;19(5):357-365.

11. Stahl SM, Morrissette DA. Stahl's Illustrated Violence: Neural Circuits, Genetics and Treatment. New York: Cambridge University Press; 2014

12. Stahl SM, Morrissette DA, Cummings M, et al. California State Hospital Violence Assessment and Treatment (Cal-VAT) guideline. CNS Spectr. 2014;19(5):449-465.

13. Stahl SM. Mechanism of action of brexpiprazole: comparison with aripiprazole. CNS Spectr. 2016;21(1):1-6.

14. Grossberg G, Kohegyi E, Mergel V, et al. Efficacy and safety of a fixed dose of brexpiprazole for the treatment of agitation in Alzheimer's type dementia: a randomized, double blind fixed dose 12 week, placebo controlled global clinical trial. Abstract presented at American Association of Geriatric Psychiatry, Honolulu, HI, March 2018.

15. Cummings J, Kohegyi E, Mergel V, et al. Efficacy and safety of flexibly dosed brexpiprazole for the treatment of agitation in Alzheimer type dementia: a randomized, double-blind, flexibly dosed 12 week placebo controlled global clinical trial. Abstract presented at American Association of Geriatric Psychiatry, Honolulu, HI, March 2018.

16. Stahl SM. Mechanism of action of dextromethorphan/quinidine: comparison with ketamine. CNS Spectr. 2013;18(5):225-227.

17. Stahl SM. Dextromethorphan-quinidine-responsive pseudobulbar affect (PBA): psychopharmacological model for wide-ranging disorders of emotional expression? CNS Spectr. 2016;21(6):419-423.

18. Porsteinsson AP, Antonsdottir IM. An update on the advancements in the treatment of agitation in Alzheimers disease. Exp Opin Pharmacother. 2017;18(6):611-620.

19. Cummings J, Lyketsos C, Tariot $P$, et al. Dextromethorphan/ quinidine (AVP-923) efficacy and safety for treatment of agitation in Alzheimer's disease: results from a phase 2 study (NCT01584440). Poster \# NR 11, American Association for Geriatric Psychiatry Annual Meeting, New Orleans, LA, March 27-30, 2015. 